\title{
Influence of Nanostructuration on PbTe Alloys Synthesized by Arc-Melting
}

\author{
Javier Gainza ${ }^{1,2}, * \mathbb{C}$, Federico Serrano-Sánchez ${ }^{1}$, Neven Biskup ${ }^{2,3}$, Norbert Marcel Nemes ${ }^{2} \mathbb{C}$, \\ José Luis Martínez ${ }^{1}{ }^{1}$, María Teresa Fernández-Díaz ${ }^{4}$ and José Antonio Alonso ${ }^{1}$ (i) \\ 1 Instituto de Ciencia de Materiales de Madrid, C.S.I.C., Cantoblanco, E-28049 Madrid, Spain; \\ fserrano@icmm.csic.es (F.S.-S.); martinez@icmm.csic.es (J.L.M.); ja.alonso@icmm.csic.es (J.A.A.) \\ 2 Departamento de Física de Materiales, Universidad Complutense de Madrid, E-28040 Madrid, Spain; \\ nbiskup@pdi.ucm.es (N.B.); nmnemes@fis.ucm.es (N.M.N.) \\ 3 Instituto Pluridisciplinar, Universidad Complutense de Madrid, E-28040 Madrid, Spain \\ 4 Institut Laue Langevin, BP 156X, Grenoble F-38042, France; ferndiaz@ill.fr \\ * Correspondence: j.gainza@csic.es
}

Received: 25 October 2019; Accepted: 14 November 2019; Published: 18 November 2019

check for updates

\begin{abstract}
PbTe}$-based alloys have the best thermoelectric properties for intermediate temperature applications (500-900 K). We report on the preparation of pristine $\mathrm{PbTe}$ and two doped derivatives $\left(\mathrm{Pb}_{0.99} \mathrm{Sb}_{0.01} \mathrm{Te}\right.$ and $\mathrm{Ag}_{0.05} \mathrm{Sb}_{0.05} \mathrm{~Pb}_{0.9} \mathrm{Te}$, so-called LAST18) by a fast arc-melting technique, yielding nanostructured polycrystalline pellets. XRD and neutron powder diffraction (NPD) data assessed the a slight Te deficiency for $\mathrm{PbTe}$, also yielding trends on the displacement factors of the $4 a$ and $4 b$ sites of the cubic $F m-3 m$ space group. Interestingly, SEM analysis shows the conspicuous formation of layers assembled as stackings of nano-sheets, with 20-30 nm thickness. TEM analysis shows intra-sheet nanostructuration on the $50 \mathrm{~nm}$ scale in the form of polycrystalline grains. Large numbers of grain boundaries are created by this nanostructuration and this may contribute to reduce the thermal conductivity to a record-low value of $1.6 \mathrm{Wm}^{-1} \mathrm{~K}^{-1}$ at room temperature. In LAST18, a positive Seebeck coefficient up to $600 \mu \mathrm{V} \mathrm{K}{ }^{-1}$ at $450 \mathrm{~K}$ was observed, contributing further towards improving potential thermoelectric efficiency.
\end{abstract}

Keywords: thermoelectrics; nanostructuration; lattice thermal conductivity; lead telluride; neutron powder diffraction

\section{Introduction}

Thermoelectric materials are relevant for a world relying on clean and sustainable energy sources. Thermoelectrics can directly convert heat into electrical energy, and their efficiency is evaluated by the figure of merit $Z T=S^{2} \sigma T / \kappa$, where $S$ is the Seebeck coefficient, $\sigma$ is the electrical conductivity, $T$ is the absolute temperature, and $\kappa$ is the total thermal conductivity, which contains the sum of the lattice $\left(\kappa_{l}\right)$ and electronic contributions $\left(\kappa_{e}\right)$ [1].

Several approaches have been developed to boost the thermoelectric performance in different ways, such as band engineering [2,3] or hierarchical architectures [4], leading to highly competitive ZT values $>1.5$ [5-8]. Among all these strategies, nanostructuration is a key role to bear in mind, because it is a useful tool to effectively reduce the thermal conductivity [4,9-11], and it has already been used in different chalcogenide-type compounds [12,13].

In recent years, tellurium-based compounds are among the most efficient thermoelectric materials, for instance, $\mathrm{Bi}_{2} \mathrm{Te}_{3}$, is widely used in near room temperature applications [1,14], or GeTe and its alloys [15-19], rare-earth tellurides [20-24], or PbTe [25-29]. PbTe-based thermoelectrics are the best performing materials in the middle-temperature range of 500 to $900 \mathrm{~K}[1,3,4,30,31]$, so they are one of 
the best options available for harvesting wasted thermal energy. In particular, Ag and Sb doping in the so-called LAST-type compounds, induce an excellent performance [30,32,33]. To further improve $\mathrm{PbTe}$ thermoelectric properties, it is important to understand the role that distinct defects may play on the lattice thermal conductivity [28], and their effects in the density of states near the bottom of the conduction band and the top of the valence band [25,29,34,35], as well as broadening our knowledge on their crystalline structures.

The first achievement of very low lattice thermal conductivities in these tellurides using nanoscale features consisted of designing superlattice structures grown by molecular beam epitaxy (MBE) [36] and chemical vapor deposition (CVD) [37]. As for other techniques, solvothermal routes have been used for nanostructuring to fabricate $\mathrm{PbTe}$ nanoboxes [38]. However, expensive and complex synthesis procedures are necessary to grow superlattices or produce nanoboxes, which make them impractical for real-world applications and devices [39]. For this reason, it is worth looking for alternative methods that lead to nanostructured and mechanically robust materials.

The effect of $\mathrm{Sb}$ or $\mathrm{Ag}$ addition in the structure of lead telluride has been studied before, concluding that the Sb-doping achieves a better thermoelectric performance [40,41], so we have synthesized this composition to be able to compare both pristine and doped samples. In the same way, $\mathrm{Ag}_{0.05} \mathrm{Sb}_{0.05} \mathrm{~Pb}_{0.9} \mathrm{Te}$ (LAST18) was proposed several years ago as an efficient thermoelectric material above room temperature [30], and it has been extensively studied since then [42-45].

We have established arc-melting as a direct procedure to synthesize highly nanostructured $\mathrm{Bi}_{2} \mathrm{Te}_{3}[46,47]$ and SnSe [48] chalcogenides in short reaction times, obtaining hard pellets that could be directly used into devices. In this way, we avoid the use of sintering techniques, such as spark plasma sintering (SPS) or hot-pressing method, making the process more straightforward and easily scalable. In this work, we describe the preparation, with the same straightforward arc-melting method, of cubic $\mathrm{PbTe}$ and $\mathrm{PbTe}(\mathrm{Sb}, \mathrm{Ag})$ specimens showing a conspicuous laminar nanostructure, responsible for a significant reduction of the thermal conductivity. The samples have been structurally studied using X-ray diffraction (XRD) and neutron powder diffraction (NPD), as well as scanning electron microscopy (SEM), transmission electron microscopy (TEM) and the three main thermoelectric properties (Seebeck coefficient, resistivity, and thermal conductivity) that were measured as a function of temperature.

\section{Materials and Methods}

The pristine $\mathrm{PbTe}$ compound and derivative $\mathrm{Pb}_{0.99} \mathrm{Sb}_{0.01} \mathrm{Te}$ and $\mathrm{Ag}_{0.05} \mathrm{Sb}_{0.05} \mathrm{~Pb}_{0.90} \mathrm{Te}$ (LAST18) alloys were synthesized in an Edmund Buhler MAM-1 mini-arc furnace (Bodelshausen, Germany), using direct arc melting in a water-cooled copper crucible, with a tungsten electrode under purified argon atmosphere. The starting materials were pure elements of $\mathrm{Pb}(99.9 \%$, Cerac, Milwaukee, WI, USA), Te (99.99\%, Alfa Aesar, Kandel, Germany), Ag (99.99\%, Cerac, Milwaukee, WI, USA), and $\mathrm{Sb}(99.5 \%$, Alfa Aesar), which were weighted and mixed according to the stoichiometric ratio. Part of the resulting ingots was ground to powder for structural characterization, and the remaining part was pressed in a Retsch Pellet Press PP25 (Haan, Germany) under an isostatic pressure of $10 \mathrm{MPa}$, and then cut with a diamond saw in bar-shape to perform transport measurements. All thermoelectric properties were measured perpendicular to the pellet pressing direction, using a Physical Properties Measurement System (PPMS) by Quantum Design (San Diego, CA, USA) in the residual vacuum of He atmosphere, under a pressure of $0.1 \mathrm{MPa}$ in the temperature range of 2 to $390 \mathrm{~K}$. In complement, high-temperature measurements were performed on the cold-pressed sample in a homemade apparatus, along the direction of the pellet pressing [49]. The Hall coefficient was measured using the four-probe resistivity option of the PPMS in delta-mode with a DC current of $\mathrm{I}=5 \mathrm{~mA}$ as a function of the magnetic field up to $\pm 8 \mathrm{~T}$. The density of the cold-pressed pellet was $\sim 96 \%$ of the theoretical crystallographic density.

Phase characterization was carried out for the pulverized sample using X-Ray diffraction (XRD) on a Bruker-AXS D8 diffractometer (Karlsruhe, Germany, $40 \mathrm{kV}, 30 \mathrm{~mA}$ ), run by DIFFRACTPLUS software (version 2.5.0, Bruker-AXS, Karlsruhe, Germany), in Bragg-Brentano reflection geometry with $\mathrm{Cu} \mathrm{K} \alpha$ radiation $(\lambda=1.5418 \AA$ ). Moreover, NPD was used to characterize the crystal structure concerning 
possible atomic vacancies and displacement factors. High-resolution patterns were collected in the D2B diffractometer at the Institut Laue-Langevin, Grenoble, France, in the high-flux configuration with a neutron wavelength $\lambda=1.549 \AA$ at $298 \mathrm{~K}$ (RT) for PbTe. Typically, $2 \mathrm{~g}$ of the samples were measured in a vanadium can. Diffraction data were analyzed using the Rietveld method employing the FULLPROF program (version Sept 2018, Grenoble, France). The line shape of the diffraction peaks was modeled by a pseudo-Voigt function. The following parameters were refined in the final run for all the atoms: scale factor, zero shift, background points, pseudo-Voigt corrected for asymmetry parameters, half-width, unit-cell parameters, and isotropic displacement factors. Occupancy factors for Te atoms were also refined from NPD data. The coherent scattering lengths of $\mathrm{Pb}$, Te, Ag, and Sb were 9.405, 5.80, 5.992, and $5.57 \mathrm{fm}$, respectively. High-resolution FE-SEM images were collected in an FEI-Nova NanoSEM 230 microscope (Denton, Texas, TX, USA).

Transmission electron microscopy (TEM) and scanning transmission electron microscopy (STEM) were carried out in a JEOL ARM 200 electron microscope (Peabody, MO, USA) with the aberration corrector enabling the spatial resolution of $0.8 \AA$ when operated at $200 \mathrm{kV}$. The microscope is equipped with a Gatan Quantum electron energy loss spectrometer (EELS, Pleasanton, CA, USA).

\section{Results and Discussion}

\subsection{Crystal Structure}

Figure 1 illustrates the Rietveld-refined XRD pattern of PbTe prepared by arc melting after carefully grinding the as-grown ingot. The diagram corresponds to a single-phase, well-crystallized $\mathrm{NaCl}$-like structure with cubic symmetry, defined in the face-centered space group $\mathrm{Fm}-3 \mathrm{~m}$, with unit-cell parameter $\mathbf{a}=6.4595(1) \AA$ A. The unit-cell size is substantially identical to that described in the literature, e.g., $6.46 \AA$ [50]. Similar diagrams were obtained for Sb-doped and LAST18 alloys.

Neutron powder diffraction (NPD) is essential to obtain accurate structural details of $\mathrm{PbTe}$ and PbTe-LAST-18. Neutrons sample a much wider range of the reciprocal space. The displacement factors can be determined precisely thanks to the lack of form factors. Furthermore, a strong aspect of this study, using NPD, is the precise characterization of any possible off-stoichiometry within the relevant crystalline phase. Losses due to evaporation are an important issue for many thermoelectrics, and NPD can keep track of the consequent stoichiometry changes, refining the occupation factor of each atom position. Despite the possible evaporation of the various elements during arc-melting, NPD showed practically the same ratio as the weighed elements before the synthesis, as described below. Technical advantages of NPD over XRD include the bulk (larger sample quantity) analysis, and the minimization of preferred orientation effects by the packing of the ground crystals in vanadium cylinders, and the rotation of the sample holder during the experiments.

The crystal structure of $\mathrm{PbTe}$ was refined in the NaCl-type, defined in the cubic Fm-3m space group [50] from NPD at RT, with Pb and Te atoms located at $4 a(0,0,0)$ and $4 b(1 / 2,1 / 2,1 / 2)$ sites, respectively. Table 1 includes the atomic parameters and displacements factors. The occupancy factors of $\mathrm{Pb}$ and $\mathrm{Te}$ could be refined and a slight Te deficiency was detected within the standard deviations $\left(f_{\text {occ }}=0.986(5)\right.$, see Table 1$)$. Regarding the PbTe-LAST18 specimen, we proposed a model corresponding to the stoichiometry $\mathrm{Ag}_{0.05} \mathrm{Sb}_{0.05} \mathrm{~Pb}_{0.90} \mathrm{Te}$ (equivalent to $\mathrm{AgPb}_{18} \mathrm{SbTe}_{20}$ ) where $\mathrm{Ag}$ and $\mathrm{Sb}$ occupy at random the $4 a$ sites together with $\mathrm{Pb}$. This model provided good agreement factors (Table 1 and Figure 1c), observing a slight Te deficiency, only two standard deviations away from the full stoichiometry. This fact additionally assessed that $\mathrm{Ag}$ and $\mathrm{Sb}$ are not located at Te sites since both elements exhibit higher scattering lengths than $\mathrm{Te}$. Remarkably, the $\mathrm{Pb}$ displacement factors are consistently large $\left(\approx 1.8 \AA^{2}\right)$, and almost double than those of Te atoms. The smearing of the nuclear scattering density of $\mathrm{Pb}$ may be related to the presence of the lone electron pair attributable to $\mathrm{Pb}^{2+}$ ions (in an ionic model for lead telluride), which for the very symmetric unit cell is certainly located at random in subsequent $\mathrm{PbTe}_{6}$ octahedra. This implies, in each case, a displacement of the $\mathrm{Pb}$ atoms and 
the $\mathrm{Pb}$-Te chemical bonds opposite to the location of the lone pair lobe. Globally, this accounts for the disorder or smearing of the scattering density, which translates into an enhanced displacement factor.
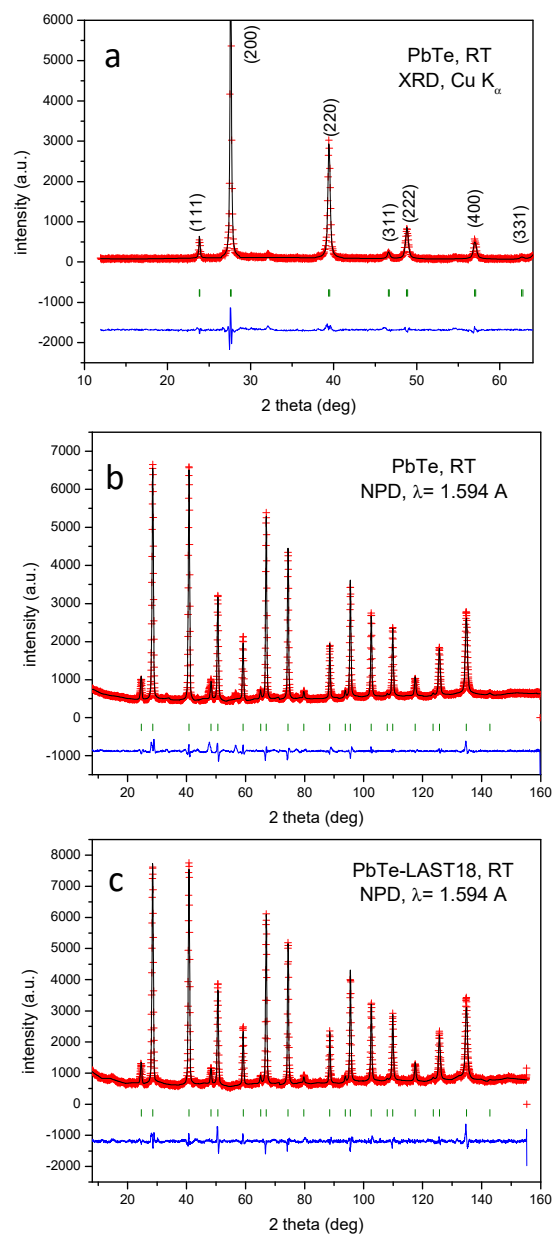

Figure 1. Observed (crosses), calculated (full line), and difference (at the bottom) profiles for (a) XRD pattern for as-grown $\mathrm{PbTe}$, Rietveld-refined in the space group $\mathrm{Fm}$ - $3 m$, (b) neutron powder diffraction (NPD) pattern for PbTe and (c) NPD patterns for PbTe-LAST18, at RT.

Figure $1 b, c$ show good agreements between observed and calculated NPD profiles for PbTe and LAST18, respectively, with correspondingly good discrepancy factors ( $R_{\text {Bragg }}=2.61$ and $2.06 \%$, respectively).

Figure 2 illustrates the crystal structure of pristine $\mathrm{PbTe}$, consisting of a simple $\mathrm{NaCl}$ arrangement of both elements in the cubic unit cell. Given the spatial positions occupied by both $\mathrm{Pb}$ and $\mathrm{Te}$, only isotropic displacement factors can be defined. Lead atoms are coordinated to six Te atoms and vice versa. The structure contains Pb-rich and Te-rich planes along the [110] crystallographic directions, which may have important repercussions on the physical properties, providing with preferred directions for the cleavage and nanostructuration yielding layered structures, thus hindering the thermal and electronic transport across the layers, as described below. 
Table 1. Structural parameters of pristine PbTe and LAST-18 $\left(\mathrm{AgPb}_{18} \mathrm{SbTe}_{20}\right)$ from NPD data at $298 \mathrm{~K}$, with $\lambda=1.594 \AA$.

\begin{tabular}{ccc}
\hline & PbTe & LAST-18 \\
\hline $\mathrm{a}(\AA)$ & $6.4595(1)$ & $6.4590(1)$ \\
\hline $\mathrm{V}\left(\AA^{3}\right)$ & $269.522(8)$ & $269.460(9)$ \\
\hline \multicolumn{3}{c}{$P b, A g, S b 4 a(0,0,0)$} \\
\hline $\mathrm{f}_{\mathrm{occ}} \mathrm{Pb} / \mathrm{Ag} / \mathrm{Sb}$ & $1.00 / 0.0 / 0.0$ & $0.90 / 0.05 / 0.05$ \\
\hline $\mathrm{B}\left(\AA^{2}\right)$ & $1.80(3)$ & $1.75(4)$ \\
\hline \multicolumn{3}{c}{$\mathrm{Te} 4 b(1 / 2,1 / 2,1 / 2)$} \\
\hline $\mathrm{f}_{\mathrm{occ}} \mathrm{Te}$ & $0.985(6)$ & $0.96(1)$ \\
\hline $\mathrm{B}\left(\AA^{2}\right)$ & $1.19(4)$ & $1.13(5)$ \\
\hline $\mathrm{R}_{\mathrm{p}}(\%)$ & Agreement factors \\
\hline $\mathrm{R}_{\mathrm{wp}}(\%)$ & 3.07 & 3.66 \\
\hline $\mathrm{R}_{\mathrm{Bragg}}(\%)$ & 4.67 & 4.95 \\
\hline$\chi^{2}$ & 8.61 & 3.04 \\
\hline & Distances $(\AA)$ \\
\hline $\mathrm{Pb}-\mathrm{Te}(\mathrm{x} 6)$ & $3.230(1)$ & $3.229(1)$ \\
\hline
\end{tabular}

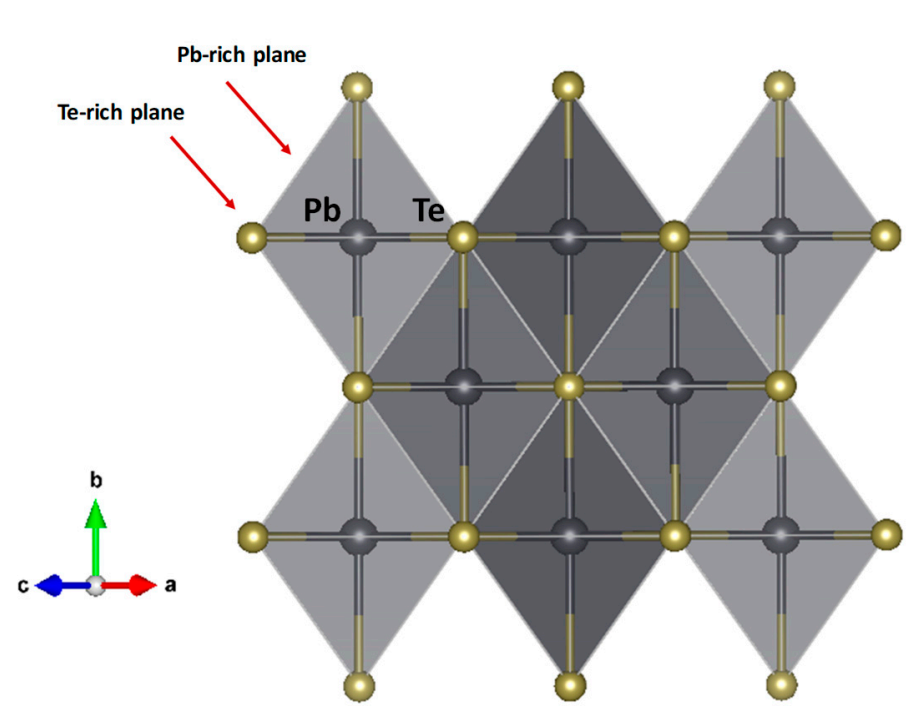

Figure 2. Crystal structure of $\mathrm{PbTe}$ along [110] directions, highlighting Te-rich planes alternating with $\mathrm{Pb}$-rich planes.

\subsection{Nanostructuration}

Figure 3 displays the morphology of the as-grown PbTe pellets imaged with several magnifications (from $5000 \times$ to $80,000 \times$ ) by field-effect-scanning electron microscopy (FE-SEM). The as-grown ingots are formed of parallel stacked nanosized sheets, also showing growing steps, as a conspicuous feature in all the examined specimens obtained by arc-melting, from various regions of various samples. Despite the 3D-type crystal structure exhibited by these materials, a strong cleavage effect is apparent, as a consequence of weak bonding directions occurring within the crystal structure, as commented above. The approximate thickness of individual sheets can be estimated in the 20 to $30 \mathrm{~nm}$ range. We consider that this lamellar nanostructuring is present throughout the volume of the material, since the images of Figure 3, and many similar, were taken from different parts of the broken-up ingot. In fact, 
according to [51], a faster cooling rate provides more homogeneous PbTe and LAST samples. Indeed, arc-melting quenches the samples from the molten state to room temperature at extremely high cooling rates estimated to be several hundreds of degrees per second.
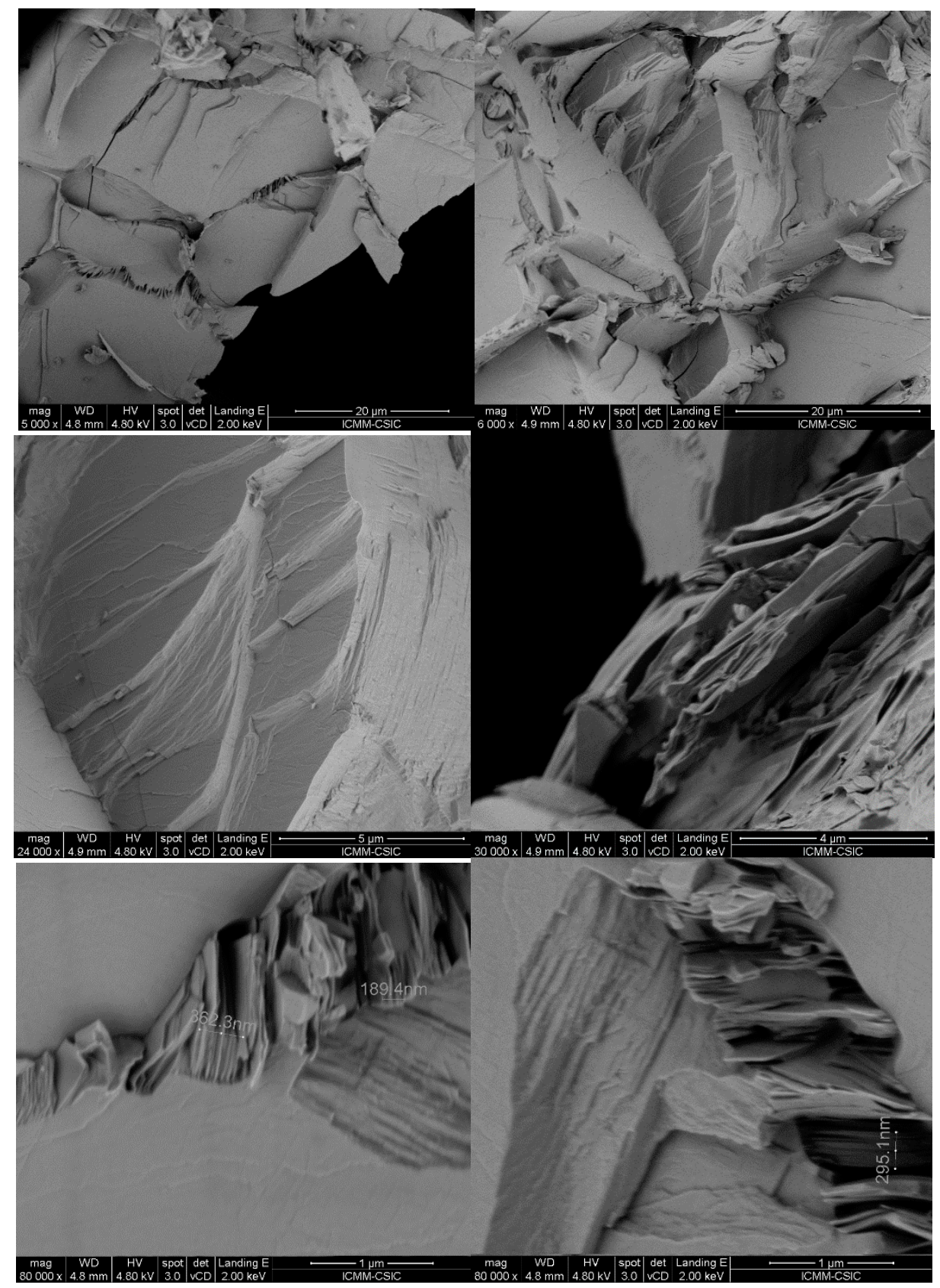

Figure 3. FE-SEM images displaying the superficial morphology of PbTe. A conspicuous laminar nanostructuration is achieved with the arc melting technique.

We have examined the crystalline powder of our $\mathrm{PbTe}$ material with transmission electron microscopy (TEM). Figure 4a shows the TEM image of a submicron-size conglomerate. The electron energy loss spectrum (EELS, Figure 4c) shows both Te and $\mathrm{Pb} \mathrm{M}_{4,5}$ absorption edges and yields 50:50 relative concentrations of both atoms. No impurities are detected by EELS, demonstrating the high purity of our material (Figure 4c). The central part of the conglomerate in Figure 4a is a single crystalline grain, as can be seen by examining diffraction patterns in different areas (inset). However, even on the scale of this image (50-100 nm), grains with different orientations can be seen: two such crystalline 
grain-boundaries are indicated by yellow dashed lines, and the different grains are labeled from 1 to 3. In the high magnification STEM ABF image (Figure 4b), one can see more closely one of these grain boundaries (marked by the arrow). One can conclude that our PbTe material is polycrystalline, even at a scale of $50 \mathrm{~nm}$.
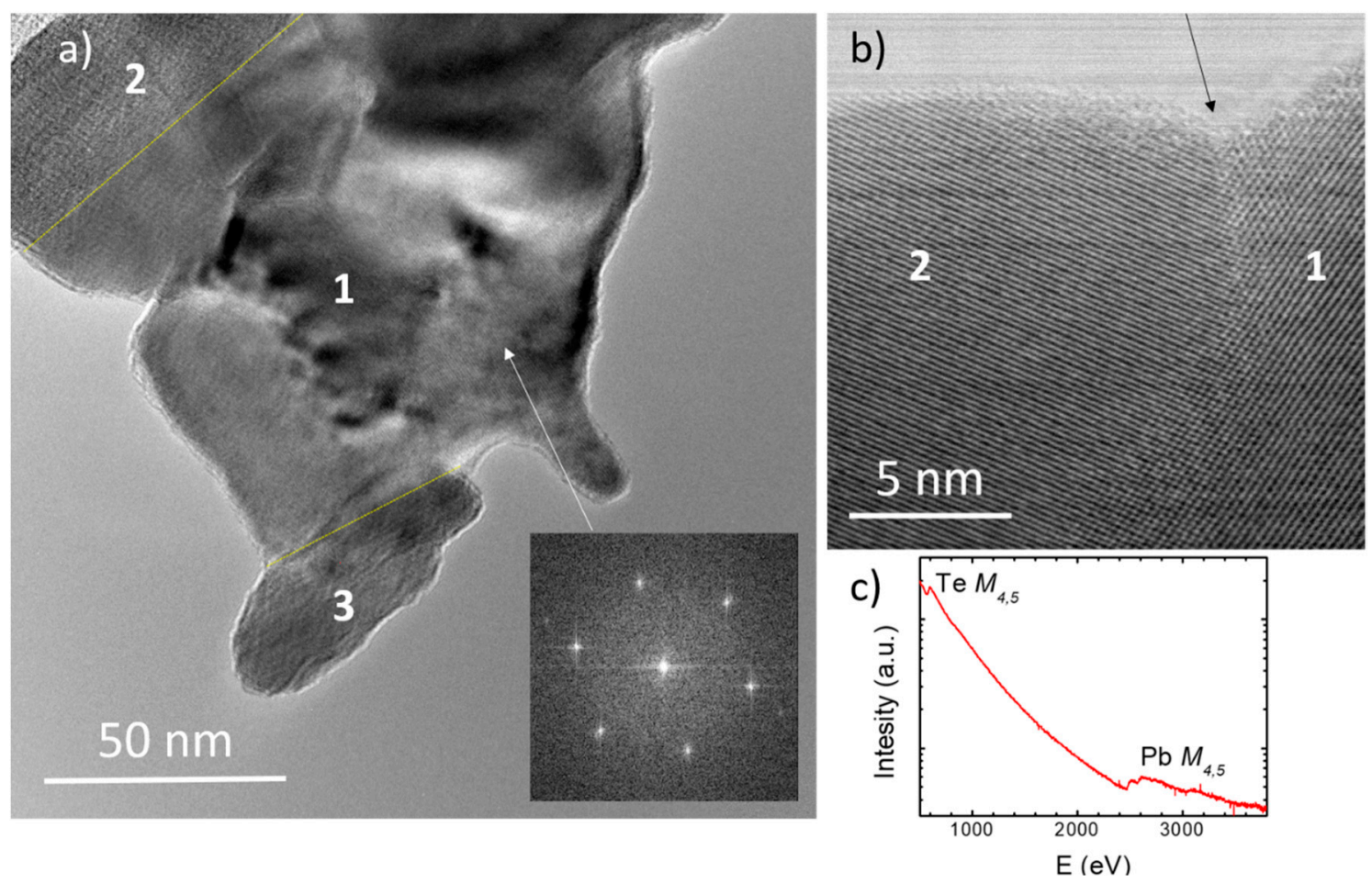

Figure 4. (a) TEM image of a conglomerate consisting of three crystalline grains. The boundaries of different grains are marked by yellow lines. The grain orientations are checked by spatially resolved diffraction patterns, the grain 1 being oriented along the [111] zone axis (inset). (b) High magnification image of the boundary between the grains 1 and 2. (c) Electron energy loss spectrometer (EELS) spectrum of the conglomerate in this figure. Only $\mathrm{Te} M_{4,5}$ and $\mathrm{Pb} M_{4,5}$ edges are detected.

This extremely small size of crystalline order may contribute to the observed low thermal conductivity, due to increased phonon scattering on a larger density of grain boundaries provided by the morphology of many nanosized sheets, produced by arc-melting.

The morphology of the PbTe based materials prepared by arc-melting is quite complex. Primarily, there are interlayer boundaries at the surfaces between neighboring nanosized sheets, which are clearly shown in the SEM images of Figure 3. Furthermore, as the high-resolution TEM images along the [111] axis shown in Figure 4 demonstrate, each nanometric layer is made of polycrystalline material that consists of single crystalline grains of $50 \mathrm{~nm}$ characteristic size. Thus, there are two types of nanostructuring present, both on the $20-50 \mathrm{~nm}$ scale. This morphology has not been reported before in $\mathrm{PbTe}$ specimens prepared by standard procedures (ball milling, reactions in sealed quartz capsules, etc.). Note that we use the term "grain boundary" quite generally in this study to refer to both the nanosheet-to-nanosheet interfaces (Figure 3), as well as the intra-sheet boundaries between single-crystalline grains (Figure $4 b$ ).

\subsection{Transport Properties}

The electrical resistivity and the Seebeck coefficient for $\mathrm{PbTe}, \mathrm{Pb}_{0.99} \mathrm{Sb}_{0.01} \mathrm{Te}$, and $\mathrm{AgPb}_{19} \mathrm{SbTe}_{20}$ are displayed in Figure 5; the corresponding curves for LAST18 data at mid-temperature are displayed in Figure 6. The resistivity of the pristine $\mathrm{PbTe}$ and $\mathrm{Pb}_{0.99} \mathrm{Sb}_{0.01} \mathrm{Te}$ increased with temperature in the 2-390 K range, as expected for metallic compounds; that of the Sb-doped sample was slightly lower, 
and the difference increased with temperature. For instance, at $300 \mathrm{~K}$ PbTe exhibited a resistivity of $1.6 \times 10^{-4} \Omega \cdot \mathrm{m}$ at $300 \mathrm{~K}$, whereas for the Sb-doped compound it was $6.5 \times 10^{-5} \Omega \cdot \mathrm{m}$. It seems that the $\mathrm{Sb}$ doping increased the electron concentration, raising the electrical conductivity while the Seebeck coefficient (Figure $5 b$ ) was reduced (in absolute value). This behavior has been already reported in Sb-doped PbTe prepared by a melting procedure in vacuum sealed-tubes $[41,52]$.

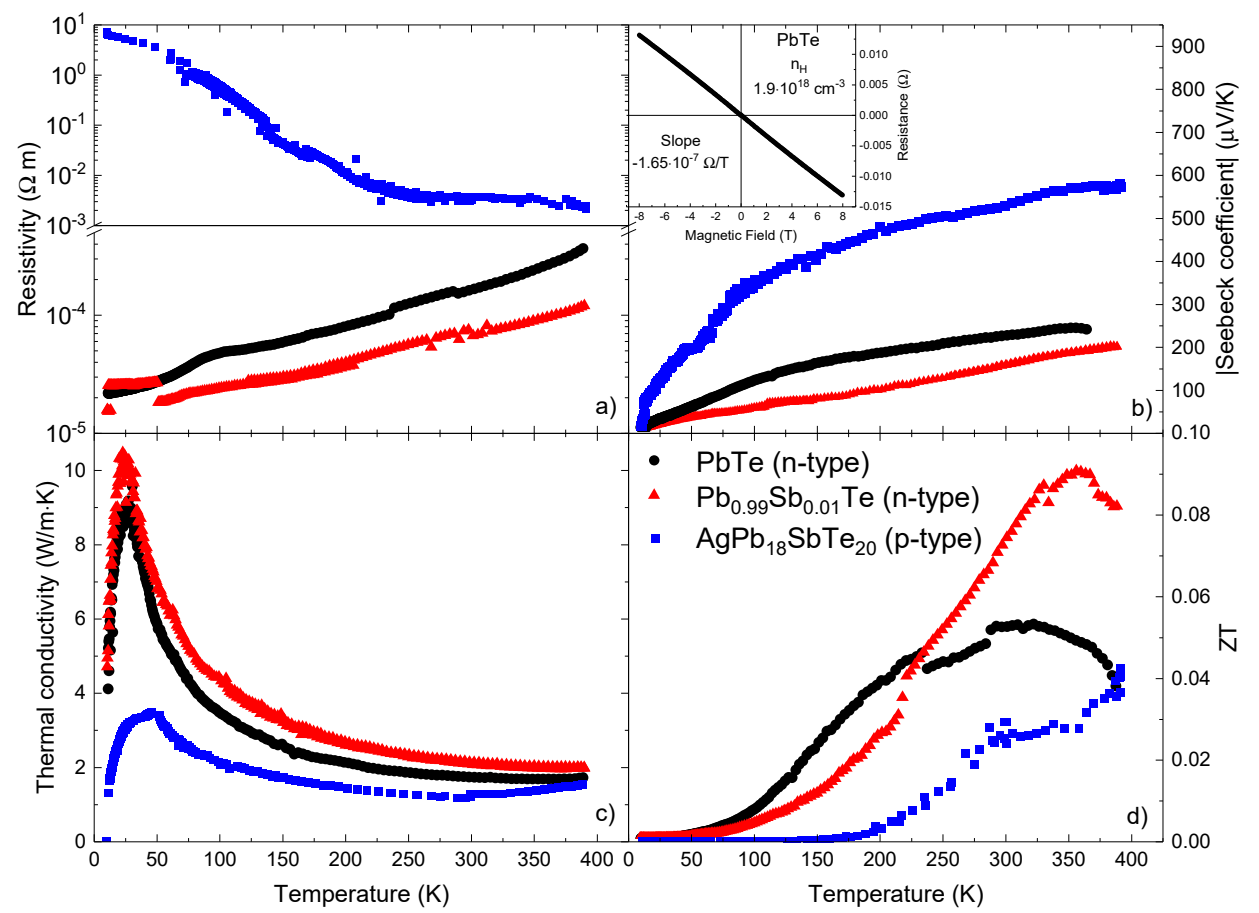

Figure 5. (a) Resistivity, (b) Seebeck coefficient, (c) thermal conductivity, and (d) figure of merit versus temperature for $\mathrm{PbTe}, \mathrm{Pb}_{0.99} \mathrm{Sb}_{0.01} \mathrm{Te}$, and LAST18 prepared by arc melting. The inset in (c) shows the Hall Effect measurement of the pristine $\mathrm{PbTe}$.

The behavior of the Seebeck coefficient from $10 \mathrm{~K}$ up to $390 \mathrm{~K}$ displayed in Figure $5 \mathrm{~b}$ is similar to those reported elsewhere $[2,25,53,54]$, where the thermopower is slowly but continuously rising up to higher temperatures. At room temperature, the Seebeck coefficient of $\mathrm{PbTe}$ is reported to be $150 \mu \mathrm{V} / \mathrm{K}$ [53], or even below that value [26], like the heavy hole-doped PbTe, which shows a Seebeck coefficient around $50 \mu \mathrm{V} / \mathrm{K}$ [54]. Compared with these data, the Seebeck coefficients of our samples were higher (in absolute value), reaching $-220 \mu \mathrm{V} / \mathrm{K}$ at RT, which is also related to the higher resistivity we found, following the well-known Pisarenko relationship. The antimony doping in this sample did not alter the fact that the electrons are the majority carriers, yielding an n-type material, in accordance with the results for other $\mathrm{Sb}$ doping levels [40].

At room temperature, the Seebeck coefficient should be positive and then switch to a negative value with increasing temperature $[55,56]$. We think that the obtained behavior of the Seebeck coefficient for pristine PbTe could occur due to slight evaporation of Te during synthesis. We can identify the tiny reflection on $\mathrm{XRD}$ at $\sim 32^{\circ}$ as $\mathrm{Pb}$ metal. Therefore, the material could probably have a slight deviation from stoichiometry, as shown from NPD data, and also contain precipitates of $\mathrm{Pb}$. That is likely the reason why the $\mathrm{PbTe}$ material shows n-type behavior at $300 \mathrm{~K}$. The room temperature Hall Effect measurements (inset of Figure 5c) confirmed the n-type behavior of our pristine $\mathrm{PbTe}$, showing a carrier concentration of $\sim 1.9 \times 10^{18} \mathrm{~cm}^{-3}$, similar to other reported results [40], but a bit lower than the optimum carrier concentration for n-type lead telluride, which stands between $4-40 \times 10^{18} \mathrm{~cm}^{-3}[35,57]$. Considering a single parabolic band approximation and a scattering of charge carriers dominated by acoustic phonons [58], we calculated an effective mass of $0.19 \mathrm{~m}_{\mathrm{e}}$, 
which agrees with similar reported data for n-type lead telluride, with effective masses slightly above $0.20 \mathrm{~m}_{\mathrm{e}}$ [35].

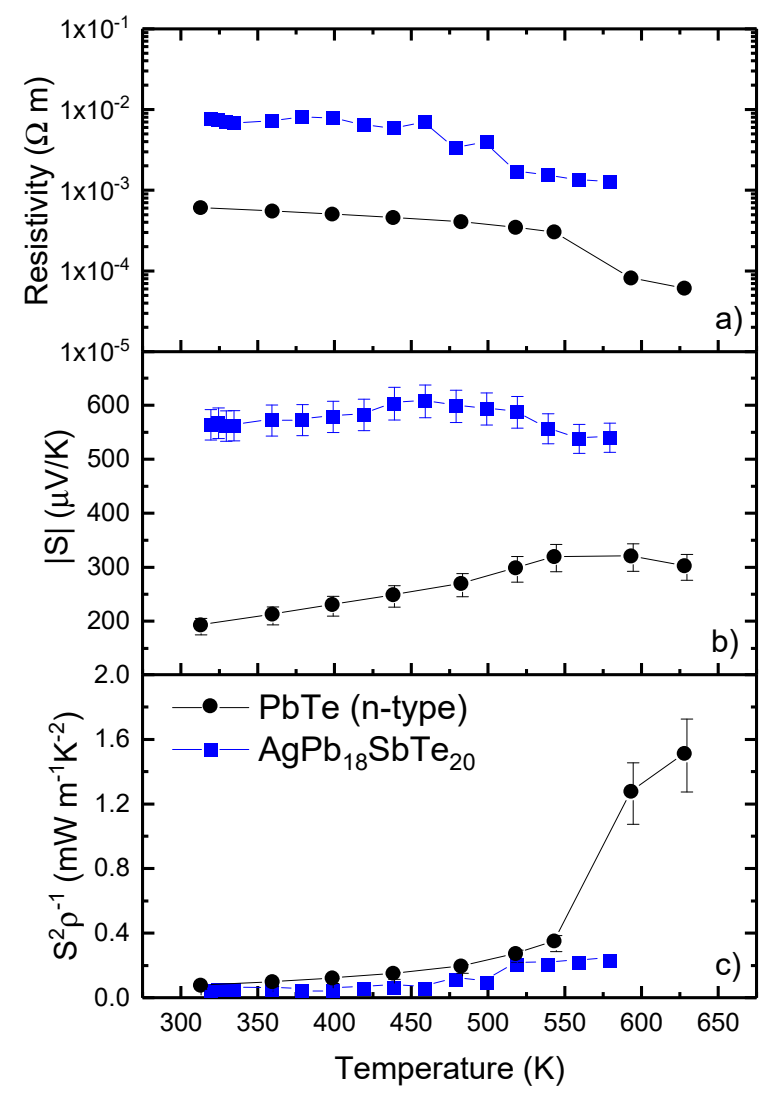

Figure 6. (a) Resistivity, (b) Seebeck coefficient, and (c) power factor above room temperature for PbTe and $\mathrm{AgPb}_{18} \mathrm{SbTe}_{20}$ prepared by arc melting.

From carrier density and resistivity, we can calculate the mobility of the electrons in $\mathrm{PbTe}$ at room temperature. This calculation showed a mobility of $205 \mathrm{~cm}^{2} / \mathrm{V} \cdot \mathrm{s}$, which was relatively high, but it is still far from the best results obtained for this composition. For example, Dow et al. reported a mobility of $2955 \mathrm{~cm}^{2} / \mathrm{V} \cdot \mathrm{s}$ for a carrier density of $2.67 \times 10^{18} \mathrm{~cm}^{-3}$ [40], and Pei et al. found a mobility above $2000 \mathrm{~cm}^{2} / \mathrm{V} \cdot \mathrm{s}$ for a carrier concentration of $4.3 \times 10^{18} \mathrm{~cm}^{-3}$ [35].

Figure $5 \mathrm{c}$ displays temperature-dependent total thermal conductivity. It shows the expected Umklapp maximum at $30 \mathrm{~K}$ and then a monotonous decrease to a minimum value of $1.6 \mathrm{~W} \mathrm{~m}^{-1} \mathrm{~K}^{-1}$ at room temperature for pristine $\mathrm{PbTe}$. For the $\mathrm{Sb}$-doped specimen, a slightly higher value was observed. Because of its high resistivity, the electronic contribution to the total thermal conductivity was negligible, so we can consider the lattice and the total thermal conductivity nearly equal. In contrast, other authors reported measurements of the thermal conductivity around $2 \mathrm{~W} \mathrm{~m}^{-1} \mathrm{~K}^{-1}[35,54]$, for PbTe and some different alloys [53,59].

The observed reduction of the thermal conductivity with respect to the literature values could be related to the nanostructuration that was observed in the FE-SEM and the TEM images. The underlying reason for this decrease is likely the strong phonon scattering happening at the grain boundaries, associated with the sheet-type nanostructuration, which is of great importance when considering the formation of nanostructures to obtain higher figures of merit [60].

This thermal conductivity can be a good starting point to improve the thermoelectric performance of nanostructured arc-melted PbTe. It has been demonstrated that PbTe alloys, such as PbTe: $\mathrm{Na}$ [54], $\mathrm{Pb}_{1-x} \mathrm{La}_{x} \mathrm{Te}$ [35], $\mathrm{Pb}_{1-\mathrm{x}} \mathrm{Mn}_{\mathrm{x}} \mathrm{Te}$ [26], PbTe-MgTe [25] or PbTe-SrTe [59], can reach a high thermoelectric performance through the improvement in their power factor. Bearing this in mind, with an adequate 
doping element, a nanostructured arc-melted $\mathrm{PbTe}$ with a great thermoelectric performance could be produced using this fast and straightforward method.

The figure of merit of both $\mathrm{PbTe}$ and $\mathrm{Pb}_{0.99} \mathrm{Sb}_{0.01} \mathrm{Te}$ is shown in Figure $5 \mathrm{~d}$. The antimony doping enhanced the thermoelectric performance of $\mathrm{PbTe} . \mathrm{Pb}_{0.99} \mathrm{Sb}_{0.01} \mathrm{Te}$ had a higher figure of merit than that of the pristine compound at room temperature, reaching almost 0.1 . This value is similar to others reported for PbTe and PbTe-based compounds at this temperature [10,28,50,52,53]. Pei et al. [35] found a ZT of 0.3 at room temperature for PbTe for a slightly higher carrier density of $4.3 \times 10^{18} \mathrm{~cm}^{-3}$, the difference with this work is mainly the high mobility they achieve in their samples, reaching a mobility of around $2000 \mathrm{~cm}^{2} / \mathrm{V} \cdot \mathrm{s}$ at room temperature.

The behavior of the resistivity and the Seebeck coefficient for PbTe and LAST18 $\left(\mathrm{AgPb}_{18} \mathrm{SbTe}_{20}\right)$ above room temperature are displayed in Figure $6 \mathrm{a}, \mathrm{b}$. The resistivity of the LAST compound decreased with temperature from $300 \mathrm{~K}$ up to $550 \mathrm{~K}$, being slightly above, but with similar behavior, than those also found by other authors [32,33]. In the case of PbTe, its resistivity stood below $1 \times 10^{-3} \Omega \cdot \mathrm{m}$ from room temperature up to $550 \mathrm{~K}$. For pristine $\mathrm{PbTe}$, the Seebeck coefficient continued increasing (in absolute value) with temperature, with the same behavior as shown below $300 \mathrm{~K}$. However, for the LAST compound, the Seebeck coefficient was positive, reaching a maximum of $620 \mu \mathrm{VK}^{-1}$ at $480 \mathrm{~K}$, and $540 \mu \mathrm{VK}^{-1}$ at $580 \mathrm{~K}$, which is significantly larger than other thermoelectric factors reported elsewhere [30,32,33], both $\mathrm{p}$ and $\mathrm{n}$-type. There have been other attempts to substitute silver by isoelectronic copper in this composition using different synthesis routes [61], yielding similar transport properties than that of LAST-18. However, the resulting thermopower is almost $50 \%$ lower, especially at higher temperatures, which results in a lower figure of merit compared with the $\mathrm{AgPb}_{18} \mathrm{SbTe}_{20}$ composition.

The power factor for PbTe and LAST18 is shown in Figure $6 c$, calculated as $\mathrm{S}^{2} / \rho$ using the experimental values of the resistivity and the Seebeck coefficient. Beyond $500 \mathrm{~K}$, the power factor increased, reaching 0.33 and $0.23 \mathrm{~mW} \mathrm{~m}^{-1} \mathrm{~K}^{-2}$ for the pristine PbTe and LAST compound, respectively, indicating promising properties in this temperature range, which is where these PbTe-type compounds present their best transport properties $[28,35,54]$. Moreover, according to literature, a minimum deviation from the stoichiometric composition can significantly alter the thermoelectric properties [32, 33], so the thermoelectric performance of LAST material could be improved, modifying the silver and lead quantity. For a more thorough comparison, data of PbTe prepared by different methods are displayed in Table 2.

Usually, the thermal conductivity of these lead telluride materials shows a monotonous decrease beyond room temperature. This allows us to calculate a lower limit for the figure of merit of this material, using the thermal conductivity at room temperature $(1.6 \mathrm{~W} / \mathrm{mK}$ and $1.28 \mathrm{~W} / \mathrm{mK}$ for PbTe and LAST, respectively) as well as the power factor for each composition. We obtained a figure of merit of $\mathrm{ZT} \sim 0.59$ at $630 \mathrm{~K}$ for $\mathrm{PbTe}$, quite higher than other reported data for pristine $\mathrm{PbTe}[55,56]$, but, of course, far from the highest figures of merit reached by other doped lead tellurides. In the case of the LAST compound, the calculated figure of merit was around ZT 0.1 at $580 \mathrm{~K}$, quite lower compared with the best results reported for LAST compounds [30,32].

$\mathrm{PbTe}$ prepared by arc-melting shows the lowest thermal conductivity and the highest Seebeck coefficient at room temperature, although its resistivity is significantly higher compared to other synthesis routes. However, the resistivity and Seebeck coefficient can be balanced with adequate doping to enhance the general thermoelectric performance. In conjunction with the short synthesis time of arc-melting, this could result in lead telluride derivatives competitive with those synthesized by other methods. 
Table 2. Comparison of lead telluride thermoelectric properties obtained by different synthesis processes. All data are measured at $300 \mathrm{~K}$.

\begin{tabular}{|c|c|c|c|c|c|c|c|}
\hline & $\begin{array}{c}\text { Thermal } \\
\text { Conductivity } \\
\left(\mathrm{W} / \mathrm{m}^{-1} \mathrm{~K}^{-1}\right)\end{array}$ & $\begin{array}{c}\text { Seebeck } \\
\text { Coefficient } \\
(\mu \mathrm{V} / \mathrm{K})\end{array}$ & $\begin{array}{l}\text { Resistivity } \\
\quad(\Omega \mathrm{m})\end{array}$ & Synthesis Method & Synthesis Time & Density & Reference \\
\hline \multirow[t]{2}{*}{$\begin{array}{c}\text { PbTeM } \\
\text { (This Work) } \\
\left(\mathrm{n}=1.9 \times 10^{18}\right)\end{array}$} & 1.6 & -215 & $1 \times 10^{-4}$ & $\begin{array}{l}\text { Arc-melting + } \\
\text { cold pressing }\end{array}$ & $\sim 1 \mathrm{~h}$ & $\sim 96 \%$ & - \\
\hline & 2.25 (Klattice $)$ & - & - & $\begin{array}{l}\text { Quartz tubes (Liquid } \\
\text { matrix encapsulation) }\end{array}$ & Several hours & - & [13] \\
\hline \multirow[t]{2}{*}{$\mathrm{PbTe}$} & 4.25 & 30 & $4.2 \times 10^{-6}$ & Quartz tubes + SPS & $\sim 60 \mathrm{~h}$ & - & {$[62]$} \\
\hline & 3.8 & 60 & $4 \times 10^{-6}$ & $\begin{array}{l}\text { Graphite coated } \\
\text { quartz tubes }\end{array}$ & $\sim 62 \mathrm{~h}$ & - & [10] \\
\hline $\begin{array}{c}\text { PbTe } \\
\left(\mathrm{n}=4.3 \times 10^{18}\right)\end{array}$ & 2.1 & -170 & $1.2 \times 10^{-5}$ & \multirow{2}{*}{$\begin{array}{l}\text { Graphite coated quartz } \\
\text { tubes + Hot pressing }\end{array}$} & \multirow{2}{*}{$\begin{array}{l}8 \mathrm{~h}+3 \text { days } \\
\text { of annealing }\end{array}$} & $>98 \%$ & \multirow[t]{2}{*}{ [35] } \\
\hline $\begin{array}{c}\text { PbTe } \\
\left(\mathrm{n}=9.4 \times 10^{19}\right)\end{array}$ & 5.5 & -40 & $1 \times 10^{-6}$ & & & $>98 \%$ & \\
\hline $\begin{array}{c}\text { PbTe } \\
\text { Nanocrystals }\end{array}$ & 1.85 & 150 & $8 \times 10^{-3}$ & $\begin{array}{l}\text { Low temperature route } \\
(353 \mathrm{~K}) \text { in a micellar } \\
\text { medium + SPS }\end{array}$ & $\sim 3.5 \mathrm{~h}$ & $\sim 98.5 \%$ & [63] \\
\hline
\end{tabular}




\section{Conclusions}

Three thermoelectric materials were prepared by a straightforward arc-melting technique: pristine $\mathrm{PbTe}, \mathrm{Pb}_{0.99} \mathrm{Sb}_{0.01} \mathrm{Te}$, and $\mathrm{Ag}_{0.05} \mathrm{Sb}_{0.05} \mathrm{~Pb}_{0.9} \mathrm{Te}$ (LAST18). All show highly nanostructured morphology with improved thermal transport properties. A structural NPD study accurately determined the displacement factors at the $\mathrm{NaCl}$-type structure and assessed the almost full stoichiometry of the specimens, with a slight Te deficiency for PbTe. The trend to cleave and to form nanostructured materials, despite the 3D crystal structure, is observed by FE-SEM. TEM imaging revealed intra-sheet nanostructuration on a $50 \mathrm{~nm}$ scale. This nanostructuring affects the physical properties of the material, enhancing the phonon scattering and yielding a reduced thermal conductivity with respect to literature values, on samples prepared by alternative procedures. The as-grown robust pellets are suitable to be manipulated and used in thermoelectric devices, and their physical properties could be improved even more with proper doping elements.

Author Contributions: Conceptualization, F.S.-S., J.L.M., and J.A.A.; Data curation, J.G., F.S.-S., N.B., and M.T.F.-D.; Formal analysis, J.G., N.M.N., and J.A.A.; Funding acquisition, J.L.M. and J.A.A.; Investigation, J.G. and F.S.-S.; Methodology, F.S.-S., N.M.N., N.B., M.T.F.-D., and J.A.A.; Resources, M.T.F.-D.; Software, J.G.; Supervision, J.L.M., M.T.F.-D., and J.A.A.; Validation, J.A.A.; Writing-original draft, J.G., and J.A.A.; Writing-review and editing, J.G., F.S.-S., N.B., N.M.N., J.L.M., M.T.F.-D., and J.A.A.

Funding: This research was funded by the Spanish Ministry of Science, Innovation and Universities for granting the project MAT2017-84496-R and RTI2018-097895-B-C43.

Acknowledgments: We are grateful to the Spanish Ministry of Science, Innovation and Universities (MICIIN) for granting the projects MAT2017-84496-R and RTI2018-097895-B-C43, and to ILL for making all facilities available for the neutron diffraction experiments. J.G. thanks MICINN for granting the FPI contract PRE2018-083398. TEM measurements were performed in the Centro Nacional de Microscopía Electrónica (CNME) at the Universidad Complutense de Madrid (UCM).

Conflicts of Interest: The authors declare no competing interests, financial or otherwise.

\section{References}

1. Snyder, G.J.; Toberer, E.S. Complex thermoelectric materials. Nat. Mater. 2008, 7, 105-114. [CrossRef]

2. Pei, Y.; Wang, H.; Snyder, G.J. Band engineering of thermoelectric materials. Adv. Mater. 2012, 24, 6125-6135. [CrossRef] [PubMed]

3. Pei, Y.; Shi, X.; LaLonde, A.; Wang, H.; Chen, L.; Snyder, G.J. Convergence of electronic bands for high performance bulk thermoelectrics. Nature 2011, 473, 66-69. [CrossRef] [PubMed]

4. Biswas, K.; He, J.; Blum, I.D.; Wu, C.I.; Hogan, T.P.; Seidman, D.N.; Dravid, V.P.; Kanatzidis, M.G. High-performance bulk thermoelectrics with all-scale hierarchical architectures. Nature 2012, 489, 414-418. [CrossRef] [PubMed]

5. He, W.; Wang, D.; Wu, H.; Xiao, Y.; Zhang, Y.; He, D.; Feng, Y.; Hao, Y.-J.; Dong, J.-F.; Chetty, R.; et al. High thermoelectric performance in low-cost SnS 0.91 Se 0.09 crystals. Science 2019, 365, 1418-1424. [CrossRef]

6. Chang, C.; Wu, M.; He, D.; Pei, Y.; Wu, C.-F.; Wu, X.; Yu, H.; Zhu, F.; Wang, K.; Chen, Y.; et al. 3D charge and 2D phonon transports leading to high out-of-plane ZT in n-type SnSe crystals. Science 2018, 360, 778-783. [CrossRef]

7. Imasato, K.; Kang, S.D.; Snyder, G.J. Exceptional thermoelectric performance in Mg 3 Sb 0.6 Bi 1.4 for low-grade waste heat recovery. Energy Environ. Sci. 2019, 12, 965-971. [CrossRef]

8. Imasato, K.; Kang, S.D.; Ohno, S.; Snyder, G.J. Band engineering in $\mathrm{Mg}_{3} \mathrm{Sb}_{2}$ by alloying with $\mathrm{Mg} 3 \mathrm{Bi} 2$ for enhanced thermoelectric performance. Mater. Horizons 2018, 5, 59-64. [CrossRef]

9. Kanatzidis, M.G. Nanostructured thermoelectrics: The new paradigm? Chem. Mater. 2010, 22, $648-659$. [CrossRef]

10. Biswas, K.; He, J.; Zhang, Q.; Wang, G.; Uher, C.; Dravid, V.P.; Kanatzidis, M.G. Strained endotaxial nanostructures with high thermoelectric figure of merit. Nat. Chem. 2011, 3, 160-166. [CrossRef]

11. Vaqueiro, P.; Powell, A.V. Recent developments in nanostructured materials for high-performance thermoelectrics. J. Mater. Chem. 2010, 20, 9577. [CrossRef]

12. Zhao, L.D.; Dravid, V.P.; Kanatzidis, M.G. The panoscopic approach to high performance thermoelectrics. Energy Environ. Sci. 2014, 7, 251-268. [CrossRef] 
13. Sootsman, J.R.; Pcionek, R.J.; Kong, H.; Uher, C.; Kanatzidis, M.G. Strong Reduction of Thermal Conductivity in Nanostructured PbTe Prepared by Matrix Encapsulation. Chem. Mater. 2006, 18, 4993-4995. [CrossRef]

14. Witting, I.T.; Chasapis, T.C.; Ricci, F.; Peters, M.; Heinz, N.A.; Hautier, G.; Snyder, G.J. The Thermoelectric Properties of Bismuth Telluride. Adv. Electron. Mater. 2019, 5, 1800904. [CrossRef]

15. Dong, J.; Sun, F.; Tang, H.; Pei, J.; Zhuang, H.; Hu, H.; Zhang, B.; Pan, Y.; Li, J. Medium-temperature thermoelectric GeTe: vacancy suppression and band structure engineering leading to high performance. Energy Environ. Sci. 2019, 12, 1396-1403. [CrossRef]

16. Li, J.; Zhang, X.; Lin, S.; Chen, Z.; Pei, Y. Realizing the High Thermoelectric Performance of GeTe by Sb-Doping and Se-Alloying. Chem. Mater. 2017, 29, 605-611. [CrossRef]

17. Hong, M.; Zou, J.; Chen, Z. Thermoelectric GeTe with Diverse Degrees of Freedom Having Secured Superhigh Performance. Adv. Mater. 2019, 31, 1807071. [CrossRef]

18. Perumal, S.; Samanta, M.; Ghosh, T.; Shenoy, U.S.; Bohra, A.K.; Bhattacharya, S.; Singh, A.; Waghmare, U.V.; Biswas, K. Realization of High Thermoelectric Figure of Merit in GeTe by Complementary Co-doping of Bi and In. Joule 2019, 3, 2565-2580. [CrossRef]

19. Srinivasan, B.; Gellé, A.; Halet, J.-F.; Boussard-Pledel, C.; Bureau, B. Detrimental Effects of Doping Al and Ba on the Thermoelectric Performance of GeTe. Materials (Basel). 2018, 11, 2237. [CrossRef]

20. May, A.F.; Fleurial, J.-P.; Snyder, G.J. Thermoelectric performance of lanthanum telluride produced via mechanical alloying. Phys. Rev. B 2008, 78, 125205. [CrossRef]

21. May, A.F.; Singh, D.J.; Snyder, G.J. Influence of band structure on the large thermoelectric performance of lanthanum telluride. Phys. Rev. B 2009, 79, 153101. [CrossRef]

22. Gomez, S.J.; Cheikh, D.; Vo, T.; Von Allmen, P.; Lee, K.; Wood, M.; Snyder, G.J.; Dunn, B.S.; Fleurial, J.-P.; Bux, S.K. Synthesis and Characterization of Vacancy-Doped Neodymium Telluride for Thermoelectric Applications. Chem. Mater. 2019. [CrossRef]

23. Cheikh, D.; Hogan, B.E.; Vo, T.; Von Allmen, P.; Lee, K.; Smiadak, D.M.; Zevalkink, A.; Dunn, B.S.; Fleurial, J.-P.; Bux, S.K. Praseodymium Telluride: A High-Temperature, High-ZT Thermoelectric Material. Joule 2018, 2, 698-709. [CrossRef]

24. Ma, J.M.; Clarke, S.M.; Zeier, W.G.; Vo, T.; Von Allmen, P.; Jeffrey Snyder, G.; Kaner, R.B.; Fleurial, J.-P.; Bux, S.K. Mechanochemical synthesis and high temperature thermoelectric properties of calcium-doped lanthanum telluride $\mathrm{La}_{3-\mathrm{x}} \mathrm{Ca}_{\mathrm{x}} \mathrm{Te}_{4}$. J. Mater. Chem. C 2015, 3, 10459-10466. [CrossRef]

25. Zhao, L.D.; Wu, H.J.; Hao, S.Q.; Wu, C.I.; Zhou, X.Y.; Biswas, K.; He, J.Q.; Hogan, T.P.; Uher, C.; Wolverton, C.; et al. All-scale hierarchical thermoelectrics: MgTe in PbTe facilitates valence band convergence and suppresses bipolar thermal transport for high performance. Energy Environ. Sci. 2013, 6, 3346-3355. [CrossRef]

26. Pei, Y.; Wang, H.; Gibbs, Z.M.; LaLonde, A.D.; Snyder, J.G. Thermopower enhancement in Pb1-xMnxTe alloys and its effect on thermoelectric efficiency. NPG Asia Mater. 2012, 4, 1-6. [CrossRef]

27. Lalonde, A.D.; Pei, Y.; Wang, H.; Jeffrey Snyder, G. Lead telluride alloy thermoelectrics. Mater. Today 2011, 14, 526-532. [CrossRef]

28. Chen, Z.; Jian, Z.; Li, W.; Chang, Y.; Ge, B.; Hanus, R.; Yang, J.; Chen, Y.; Huang, M.; Snyder, G.J.; et al. Lattice Dislocations Enhancing Thermoelectric PbTe in Addition to Band Convergence. Adv. Mater. 2017, 29, 1606768. [CrossRef]

29. Pei, Y.; Tan, G.; Feng, D.; Zheng, L.; Tan, Q.; Xie, X.; Gong, S.; Chen, Y.; Li, J.-F.; He, J.; et al. Integrating Band Structure Engineering with All-Scale Hierarchical Structuring for High Thermoelectric Performance in PbTe System. Adv. Energy Mater. 2017, 7, 1601450. [CrossRef]

30. Hsu, K.F.; Loo, S.; Guo, F.; Chen, W.; Dyck, J.S.; Uher, C.; Hogan, T.; Polychroniadis, E.K.; Kanatzidis, M.G. Cubic AgPbmSbTe2+m: Bulk Thermoelectric Materials with High Figure of Merit. Science 2004, 303, 818-821. [CrossRef]

31. Luo, Z.-Z.; Cai, S.; Hao, S.; Bailey, T.P.; Su, X.; Spanopoulos, I.; Hadar, I.; Tan, G.; Luo, Y.; Xu, J.; et al. High Figure of Merit in Gallium-Doped Nanostructured n-Type PbTe-xGeTe with Midgap States. J. Am. Chem. Soc. 2019, 141, 16169-16177. [CrossRef] [PubMed]

32. Wang, H.; Li, J.-F.; Nan, C.-W.; Zhou, M.; Liu, W.; Zhang, B.-P.; Kita, T. High-performance $\mathrm{Ag}_{0.8} \mathrm{~Pb}_{18+x} \mathrm{SbTe}_{20}$ thermoelectric bulk materials fabricated by mechanical alloying and spark plasma sintering. Appl. Phys. Lett. 2006, 88, 092104. [CrossRef]

33. Kosuga, A.; Uno, M.; Kurosaki, K.; Yamanaka, S. Thermoelectric properties of stoichiometric $\mathrm{Ag}_{1-\mathrm{x}} \mathrm{Pb}_{18} \mathrm{SbTe}_{20}$ $(\mathrm{x}=0,0.1,0.2)$. J. Alloys Compd. 2005, 391, 288-291. [CrossRef] 
34. Ahmad, S.; Mahanti, S.D.; Hoang, K.; Kanatzidis, M.G. Ab initio studies of the electronic structure of defects in PbTe. Phys. Rev. B 2006, 74, 155205. [CrossRef]

35. Pei, Y.; Gibbs, Z.M.; Gloskovskii, A.; Balke, B.; Zeier, W.G.; Snyder, G.J. Optimum carrier concentration in n-type PbTe thermoelectrics. Adv. Energy Mater. 2014, 4, 1-12. [CrossRef]

36. Harman, T.C.; Taylor, P.J.; Walsh, M.P.; LaForge, B.E. Quantum Dot Superlattice Thermoelectric Materials and Devices. Science 2002, 297, 2229-2232. [CrossRef]

37. Venkatasubramanian, R.; Siivola, E.; Colpitts, T.; O'Quinn, B. Thin-film thermoelectric devices with high room-temperature figures of merit. Nature 2001, 413, 597-602. [CrossRef]

38. Wang, W.; Poudel, B.; Wang, D.; Ren, Z.F. Synthesis of PbTe nanoboxes using a solvothermal technique. Adv. Mater. 2005, 17, 2110-2114. [CrossRef]

39. Szczech, J.R.; Higgins, J.M.; Jin, S. Enhancement of the thermoelectric properties in nanoscale and nanostructured materials. J. Mater. Chem. 2011, 21, 4037-4055. [CrossRef]

40. Dow, H.S.; Oh, M.W.; Kim, B.S.; Park, S.D.; Min, B.K.; Lee, H.W.; Wee, D.M. Effect of Ag or Sb addition on the thermoelectric properties of PbTe. J. Appl. Phys. 2010, 108, 113709. [CrossRef]

41. Wang, S.; Yang, Z.; Sun, Y.; Xiao, Y.; Zhao, L. Synergistically optimizing charge and phonon transport properties in n-type PbTe via introducing ternary compound $\mathrm{AgSb}(\mathrm{Se}, \mathrm{Te})_{2}$. J. Alloys Compd. 2020, 815, 152463. [CrossRef]

42. Quarez, E.; Hsu, K.-F.; Pcionek, R.; Frangis, N.; Polychroniadis, E.K.; Kanatzidis, M.G. Nanostructuring, Compositional Fluctuations, and Atomic Ordering in the Thermoelectric Materials $\mathrm{AgPb}_{\mathrm{m}} \mathrm{SbTe}_{2+\mathrm{m}}$. The Myth of Solid Solutions. J. Am. Chem. Soc. 2005, 127, 9177-9190. [CrossRef] [PubMed]

43. Li, H.; Cai, K.F.; Du, Y.; Wang, H.F.; Shen, S.Z.; Li, X.L.; Wang, Y.Y.; Zhou, C.W. Preparation and thermoelectric properties of AgPb18SbTe20-xSex (x=1,2,4) materials. Curr. Appl. Phys. 2012, 12, 188-192. [CrossRef]

44. Han, M.-K.; Hoang, K.; Kong, H.; Pcionek, R.; Uher, C.; Paraskevopoulos, K.M.; Mahanti, S.D.; Kanatzidis, M.G. Substitution of $\mathrm{Bi}$ for $\mathrm{Sb}$ and its Role in the Thermoelectric Properties and Nanostructuring in $\mathrm{Ag}_{1-\mathrm{x}} \mathrm{Pb}_{18} \mathrm{MTe}_{20}$ $(\mathrm{M}=\mathrm{Bi}, \mathrm{Sb})(\mathrm{x}=0,0.14,0.3)$. Chem. Mater. 2008, 20, 3512-3520. [CrossRef]

45. Wu, J.; Yang, J.-Y.; Zhang, J.-S.; Li, G.; Peng, J.-Y.; Xiao, Y.; Fu, L.-W.; Liu, Q.-Z. Thermoelectric Properties of Sn-Substituted $\mathrm{AgPb}_{\mathrm{m}} \mathrm{SbTe}_{\mathrm{m}+2}$ via the Route of Mechanical Alloying and Plasma-Activated Sintering. J. Electron. Mater. 2012, 41, 1100-1104. [CrossRef]

46. Gharsallah, M.; Serrano-Sánchez, F.; Bermúdez, J.; Nemes, N.M.; Martínez, J.L.; Elhalouani, F.; Alonso, J.A. Nanostructured $\mathrm{Bi}_{2} \mathrm{Te}_{3}$ Prepared by a Straightforward Arc-Melting Method. Nanoscale Res. Lett. 2016, 11, 4-10. [CrossRef]

47. Gharsallah, M.; Serrano-Sanchez, F.; Nemes, N.M.; Martinez, J.L.; Alonso, J.A. Influence of Doping and Nanostructuration on n-Type $\mathrm{Bi}_{2}\left(\mathrm{Te}_{0.8} \mathrm{Se}_{0.2}\right)_{3}$ Alloys Synthesized by Arc Melting. Nanoscale Res. Lett. 2017, 12, 47. [CrossRef] [PubMed]

48. Gharsallah, M.; Serrano-Sánchez, F.; Nemes, N.M.; Mompeán, F.J.; Martínez, J.L.; Fernández-Díaz, M.T.; Elhalouani, F.; Alonso, J.A. Giant Seebeck effect in Ge-doped SnSe. Sci. Rep. 2016, 6, 1-9. [CrossRef]

49. Iwanaga, S.; Toberer, E.S.; LaLonde, A.; Snyder, G.J. A high temperature apparatus for measurement of the Seebeck coefficient. Rev. Sci. Instrum. 2011, 82, 063905. [CrossRef]

50. Korkosz, R.J.; Chasapis, T.C.; Lo, S.; Doak, J.W.; Kim, Y.J.; Wu, C.-I.; Hatzikraniotis, E.; Hogan, T.P.; Seidman, D.N.; Wolverton, C.; et al. High ZT in p-Type $(\mathrm{PbTe})_{1-2 \mathrm{x}}(\mathrm{PbSe})_{\mathrm{x}}(\mathrm{PbS})_{\mathrm{x}}$ Thermoelectric Materials. J. Am. Chem. Soc. 2014, 136, 3225-3237. [CrossRef]

51. Chen, N.; Gascoin, F.; Snyder, G.J.; Müller, E.; Karpinski, G.; Stiewe, C. Macroscopic thermoelectric inhomogeneities in $\left(\mathrm{AgSbTe}_{2}\right)_{\mathrm{x}}(\mathrm{PbTe})_{1-\mathrm{x}}$. Appl. Phys. Lett. 2005, 87, 171903. [CrossRef]

52. Srinivasan, B.; Gucci, F.; Boussard-Pledel, C.; Cheviré, F.; Reece, M.J.; Tricot, S.; Calvez, L.; Bureau, B. Enhancement in thermoelectric performance of n-type Pb-deficit Pb-Sb-Te alloys. J. Alloys Compd. 2017, 729, 198-202. [CrossRef]

53. Heremans, J.P.; Jovovic, V.; Toberer, E.S.; Saramat, A.; Kurosaki, K.; Charoenphakdee, A.; Yamanaka, S.; Snyder, G.J. Enhancement of Thermoelectric Efficiency in PbTe by Distortion of the Electronic Density of States. Science 2008, 321, 554-557. [CrossRef] [PubMed]

54. Pei, Y.; Lalonde, A.; Iwanaga, S.; Snyder, G.J. High thermoelectric figure of merit in heavy hole dominated PbTe. Energy Environ. Sci. 2011, 4, 2085-2089. [CrossRef]

55. Parashchuk, T.; Dashevsky, Z.; Wojciechowski, K. Feasibility of a high stable PbTe:In semiconductor for thermoelectric energy applications. J. Appl. Phys. 2019, 125, 245103. [CrossRef] 
56. Wang, X.-K.; Veremchuk, I.; Bobnar, M.; Zhao, J.-T.; Grin, Y. Solid solution $\mathrm{Pb}_{1-\mathrm{x}}$ Eu $\mathrm{x}$ Te: constitution and thermoelectric behavior. Inorg. Chem. Front. 2016, 3, 1152-1159. [CrossRef]

57. Xiao, Y.; Zhao, L.-D. Charge and phonon transport in PbTe-based thermoelectric materials. npj Quantum Mater. 2018, 3, 55. [CrossRef]

58. May, A.F.; Snyder, G.J. Introduction to Modeling Thermoelectric Transport at High Temperatures. In Materials, Preparation, and Characterization in Thermoelectrics; Rowe, D.M., Ed.; CRC Press: Boca Raton, FL, USA, 2012; pp. 1-18. ISBN 978-1-4398-7470-7.

59. Tan, G.; Shi, F.; Hao, S.; Zhao, L.-D.; Chi, H.; Zhang, X.; Uher, C.; Wolverton, C.; Dravid, V.P.; Kanatzidis, M.G. Non-equilibrium processing leads to record high thermoelectric figure of merit in PbTe-SrTe. Nat. Commun. 2016, 7, 12167. [CrossRef]

60. Song, Q.; Liu, T.-H.; Zhou, J.; Ding, Z.; Chen, G. Ab initio study of electron mean free paths and thermoelectric properties of lead telluride. Mater. Today Phys. 2017, 2, 69-77. [CrossRef]

61. Srinivasan, B.; Fontaine, B.; Gucci, F.; Dorcet, V.; Saunders, T.G.; Yu, M.; Cheviré, F.; Boussard-Pledel, C.; Halet, J.-F.; Gautier, R.; et al. Effect of the Processing Route on the Thermoelectric Performance of Nanostructured $\mathrm{CuPb}_{18} \mathrm{SbTe}_{20}$. Inorg. Chem. 2018, 57, 12976-12986. [CrossRef]

62. Qin, B.; Hu, X.; Zhang, Y.; Wu, H.; Pennycook, S.J.; Zhao, L. Comprehensive Investigation on the Thermoelectric Properties of p-Type PbTe-PbSe-PbS Alloys. Adv. Electron. Mater. 2019. [CrossRef]

63. Nithiyanantham, U.; Ozaydin, M.F.; Tazebay, A.S.; Kundu, S. Low temperature formation of rectangular $\mathrm{PbTe}$ nanocrystals and their thermoelectric properties. New J. Chem. 2016, 40, 265-277. [CrossRef]

(C) 2019 by the authors. Licensee MDPI, Basel, Switzerland. This article is an open access article distributed under the terms and conditions of the Creative Commons Attribution (CC BY) license (http://creativecommons.org/licenses/by/4.0/). 